\title{
Maternal weight and type 1 diabetes mellitus in children
}

Maternal BMI and weight gain during pregnancy could predict the risk of islet autoimmunity in genetically susceptible children.

Only a proportion of genetically susceptible individuals develop type 1 diabetes mellitus (T1DM), and its incidence in the population has increased over time. Despite years of research, no single environmental trigger, such as viral infection or nutrition, has been convincingly established as a causal factor for the development of T1DM.

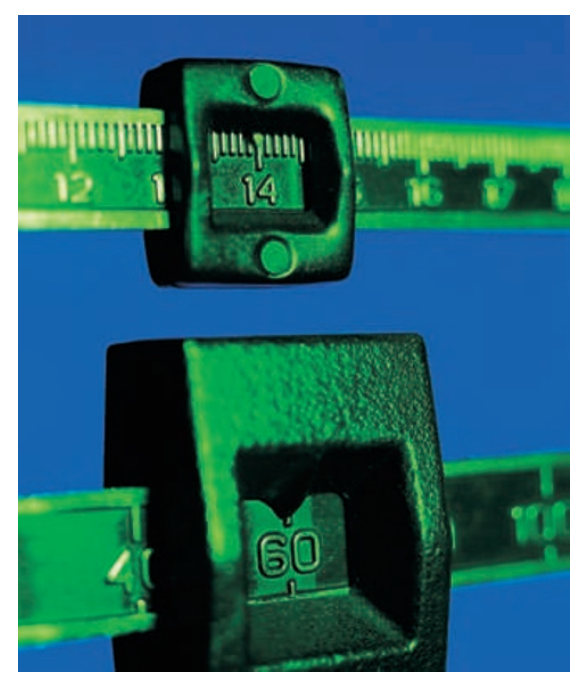

"The increase in T1DM over the past few decades has coincided with the increased prevalence of overweight in most populations, including among pregnant women," says lead author Trond Rasmussen (Norwegian Institute of Public Health, Oslo). "Despite the well-known association between maternal obesity in pregnancy and overweight in the offspring, the relation of maternal BMI or weight gain during pregnancy and the risk of T1DM or islet autoimmunity has never been studied before."

Of 46,939 Norwegian neonates genotyped in the MIDIA study, $2.14 \%$ carried the human leukocyte antigen (HLA) genotype DR4-DQ8/DR3-DQ2, which confers an approximately 20 -fold higher risk for the development of T1DM than the genotype in the general population. Blood samples from 885 children, who were positive for the HLA genotype, were collected every 3 months during the first year of life, then annually, and tested for autoantibodies against three different autoantigens: insulin, glutamic acid decarboxylase 65 (also known as glutamate decarboxylase 2) and insulinoma-associated protein 2 . Repeated positive testing for two or three islet autoantibodies is highly predictive of T1DM and occurs at a very early stage in many individuals who later develop T1DM. Maternal weight and height were assessed with questionnaires.

\section{Repeated positive testing for} two or three islet autoantibodies is highly predictive of T1DM... 77

The investigators found that both maternal BMI $\left(\geq 30 \mathrm{~kg} / \mathrm{m}^{2}\right)$ before pregnancy and a weight gain of $\geq 15 \mathrm{~kg}$ during pregnancy were prognostic of a twofold to threefold increase in the risk of islet autoimmunity.

"As opposed to many other hypotheses regarding risk factors for T1DM, maternal weight, if proved correct in future replication studies, may potentially explain the increasing incidence of T1DM over time," concludes Rasmussen.

\section{Linda Koch}

Original article Rasmussen, T. et al. Maternal BMI before pregnancy, maternal weight gain during pregnancy, and risk of persistent positivity for multiple diabetes-associated autoantibodies in children with the high-risk HLA genotype: the MIDIA study. Diabetes Care 32, 1904-1906 (2009). 